\title{
Update on the use of new anticonvulsants as mood stabilisers
}

\author{
Ian Maidment
}

\begin{abstract}
Aims and method The aim of the study was to assess and update the data related to the use of lamotrigine. gabapentin, topiramate and tiagabine as mood stabilisers. The published trials and case reports were collated from searches of the Cochrane Collaboration. Medline and Micromedex from January 1985 to January 1999. The manufacturers were also contacted.

The search included other aspects of the usage of these anticonvulsants, such as side-effects and drug interactions.

Results Most of the data are in the form of anecdotal case reports and small scale open studies. The majority of the trials lack a placebo arm and do not use standard rating scales or standard diagnostic criteria. The usefulness of the results is partly limited by the short durations and small sample sizes. Furthermore, it is often unclear whether concomitant medication was altered.

Therefore, any conclusion of effectiveness requires confirmation in double-blind placebo-controlled studies to eliminate any false-posittve response due to blas. placebo effects or random variability. There is also possibility of false-negative selection bias, as the early data tends to relate to a more refractory population. The use of these newer anticonvulsants as mood stabilisers is currently under investigation in a clinical trial programme.

Clinical implications There are no studies comparing the new anticonvulsants either with each other or with established agents. There is less evidence supporting the use of the newer anticonvulsants in blpolar disorders compared with established agents. The newer anticonvulsants should be used as third- or fourth-line agents. There is more data supporting the use of lamotrigine and gabapentin.
\end{abstract}

The second-generation anticonvulsants carbamazepine and valproate are widely used as alternative mood stabilisers to lithium. Thirdgeneration anticonvulsants such as lamotrigine and gabapentin are increasingly being used as mood stabilisers.

Lamotrigine is thought to work as an anticonvulsant by inhibiting the release of excitatory amino acid neurotransmitters, particularly glutamate and aspartate, at voltage sensitive sodium channels (Sussman, 1997). Glutamatergic abnormalities have been implicated in depression, and it is thought lamotrigine acts as a mood stabiliser by inhibiting excessive glutamate release (Sporn \& Sachs, 1997).

Gabapentin was developed originally as a structural analogue of the inhibitory neurotransmitter $\gamma$-aminobutyric acid (GABA; Sussman, 1997). It has since, however, been shown to have no significant activity at GABA receptors and its precise mode of action as an anticonvulsant is unclear (Elwes \& Binnie, 1996).

Topiramate and tiagabine are two new anticonvulsants which have been suggested to possess mood stabilisation properties. It is unclear how topiramate works, but it has been shown to enhance the activity of GABA and block the effect of glutamate (Marcotte, 1998).

\section{Clinical studies}

There are major limitations with all the reported studies. First, most of the studies are unblinded. uncontrolled, retrospective and of a limited duration. Furthermore, the treating clinician was involved in clinical assessment and standardised rating instruments were often not used. Thus, any response may have been due to patient or rater bias, spontaneous remission or a placebo effect. Second, the small samples sizes may lead to limited statistical power. Finally. because the studies are naturalistic patients usually received a wide variety of other medication including mood stabilisers and antidepressants.

Two recent articles have reviewed the use of lamotrigine in bipolar disorder (Duncan et al, 1998; Maidment, 1999). The general conclusion is that, although some initial reports are promising, there are insufficient data to recommend lamotrigine as either a first- or second-line therapy. Only one paper has subsequently been published (Calabrese et al, 1999). This study which was double-blind and placebo-controlled involved 195 patients with bipolar I disorder. 
While the study showed lamotrigine to be an effective treatment of the depressive phase, it failed to show any effectiveness in the manic phase.

In the largest currently published study gabapentin was used as a mood stabiliser in 73 patients with a diagnosis of either bipolar I or II disorder, except in two patients with a schizoaffective disorder (Ryback et al, 1997). There was a large dosage range (200-3500 mg daily). Although standardised rating scales were not used, the authors concluded that 67 patients had a positive response to gabapentin with daily activities, mood and behaviour returning to normal. The study was open, uncontrolled and retrospective. No details of concomitant medication, or how the clinician assessed patients were recorded.

In another study, 28 patients with varlous types of bipolar disorders who had not responded to standard mood stabilisers were treated with gabapentin at a mean dose of $539 \mathrm{mg}$ daily (Schaffer \& Schaffer, 1997). Some patients took other psychotropic medication including mood stabilisers.

Eighteen patients (64\%) responded according to both the psychiatrist and patient, although the criteria for a positive response were not stated. Gabapentin was discontinued in 10 patients (36\%); in two cases because of a poor response. It was discontinued in the other eight patients because of side-effects, most commonly oversedation or overactivation, although in two patients it was stopped because of an increase in rapid cycling.

A recent study reviewed the use of gabapentin in 50 patients with a mood disorder (Nassir Ghaemi et al, 1998). Only four patients received gabapentin monotherapy. The response of patients meeting DSM-IV (American Psychiatric Association, 1994) criteria for bipolar disorder or unipolar major depressive disorder was retrospectively assessed using the Clinical Global Impressions Scale for Improvement (CGI-I) (Guy, 1976).

The mean gabapentin dose was $1597 \mathrm{mg}$ daily, and the overall response rate based on an improvement in the CGI-I of at least two was $30 \%$. Only $15 \%$ of patients with unipolar depression and bipolar disorder type I responded, whereas $41 \%$ of patients with bipolar disorder type II and bipolar disorder not otherwise specified (NOS) responded. This difference, however, was not statistically significant. While noting the study's limitations, the authors concluded that gabapentin was an effective mood stabiliser, particularly in patients with a mild bipolar disorder.

There are three small scale non-blind open studies, where gabapentin was used as adjunctive therapy (Bennett et al, 1997; McElroy et al. 1997; Knoll et al, 1998). Knoll et al (1998) added gabapentin to the medication of 12 out-patients with treatment-resistant bipolar disorders. Response was assessed every 3-4 weeks using the CGI-I. The median dose used was $2400 \mathrm{mg}$ daily and the duration of treatment ranged from 3-60 weeks. Eight patients showed a marked or moderate response to gabapentin. Gabapentin was discontinued in seven patients, mainly because of excessive sedation. In conclusion, the authors stated that gabapentin was associated with a moderate improvement in mood.

Bennett et al (1997) added gabapentin to the medication of five female in-patients, three with a diagnosis of bipolar I disorder and two with a bipolar schizoaffective disorder. The dose range was 600-2400 mg daily. Based on chart review and clinical impression there was a marked response to gabapentin in three cases. These three patients received higher gabapentin doses (1500-2400 mg daily).

McElroy et al (1997) used gabapentin as an adjunctive therapy in nine patients with bipolar I or II disorder. After three months eight patients showed a moderate or marked improvement in their manic symptoms. Of these eight patients, in six cases there was a sustained antimanic response to gabapentin. In patients who responded to gabapentin the dose varied from $1600-4800 \mathrm{mg}$ every day. The authors concluded that gabapentin may have a mood stabilising effect, while noting the limitations of the trial.

Data on two unpublished trials was presented at the American Psychiatric Association 150th Annual Meeting (further details available from the author upon request). Marcotte et al (1998) retrospectively analysed the medical records of 47 patients diagnosed with bipolar disorder who received gabapentin (mean dose $1500 \mathrm{mg}$ daily) for at least six months. The majority of patients reported improvement, although the authors noted the need for double-blind, placebocontrolled trials. Young et al reported the effectiveness of gabapentin as an antidepressant in 15 patients with bipolar I or II disorder with current depression (further details available from the author upon request). There are case reports of gabapentin being used successfully to treat acute mania (Stanton et al, 1997) and behavioural dyscontrol (Ryback \& Ryback. 1995).

There are three studies investigating the use of topiramate. Marcotte et al (1998) evaluated addon topiramate therapy in 58 patients with various psychiatric disorders refractory to conventional mood stabilisers. According to DSM-IV criteria, 44 patients had a rapid cycling bipolar disorder (14 type I, six type II, seven mixed, 10 cyclothymic and seven type not specified). nine had a schizoaffective disorder, three had 
dementia and two had psychosis. The initial dose was $50 \mathrm{mg}$ a day, this was increased to a maximum of $400 \mathrm{mg}$ daily. The mean dose was $200 \mathrm{mg}$ daily (range 25-400 $\mathrm{mg}$ ), and the mean duration of treatment was 16 weeks. There was a marked or moderate improvement in the CGI-I in $62 \%$ of all patients and $52 \%$ of patients with a bipolar disorder. These results should be treated with caution because of the open, non-blind and retrospective nature of the study. Furthermore, patients were taking a variety of concurrent medication including mood stabilisers.

Calabrese et al evaluated the use of topiramate in the acute management of hospitalised treatment-refractory mania over 28 days (further details available from the author upon request). After a three-day washout period topiramate was started at a dose of $50 \mathrm{mg}$ daily and titrated upwards. Eleven patients with bipolar type I disorder received a mean dose of $313 \mathrm{mg}$ (range 50-612 mg).

Symptoms were rated with the Young Mania Rating Scale (Y-MRS; Young et al, 1978), the Brief Psychiatric Rating Scale (BPRS; Overall \& Gorham, 1962), the Hamilton Depression Rating Scale (HAM-D-17; Hamilton, 1960, 1967) and CGI-I. The Y-MRS fell from a baseline of 32 to 22 and the BPRS fell from 47 to 39 . There was no improvement in the depression rating scale. There was a marked or moderate improvement in the CGI-I in four patients (40\%). These results related to 10 patients, and it is not clear if they were significant. McElroy et al investigated the effect of topiramate in 33 patients with bipolar disorders (28 with bipolar type I, four with bipolar type II and one with a schizoaffective disorder), who did not tolerate or were unresponsive to conventional mood stabilisers (further details available from the author upon request). The topiramate dose was titrated upwards to a maximum of $1000 \mathrm{mg}$ daily. The mean duration of treatment was 123 days and the mean dose was $256 \mathrm{mg}$ daily. Patients were taking a variety of other medication including other mood stabilisers.

Based on a CGI rating modified for bipolar disorders, $52 \%$ of patients were much or very much improved. Manic symptoms appeared to respond better to topiramate; the Y-MRS decreased from 9.2 to 6.3, whereas there was a slight decrease in the Inventory of Depresstve Symptoms scale (IDS; Rush et al, 1986) from 26.3 to 23.6. There was no improvement in the Global Assessment of Functioning (GAF; Endicott et al, 1976).

The effects of tiagabine monotherapy on mood have been investigated (Dodrill et al, 1998). Sixty-six patients received low-dose tiagabine (6 mg/day), whereas 57 patients received $36 \mathrm{mg} /$ day. The study showed that the low dose was more likely to be associated with an improvement in mood.

\section{Side-effects}

Lamotrigine causes a rash in $5-10 \%$ of patients (Anonymous, 1996a). While the majority of rashes are mild and self-limiting, about $0.1 \%$ of patients develop serious skin rashes including StevensJohnson syndrome (Anonymous, 1996a). Furthermore, lamotrigine-induced fatal toxic epidermal necrolysis has been reported (Sterker et al, 1995). Factors associated with an increased risk include concomitant valproate use, high initial doses and rapid dose escalation (Sussman, 1997).

Gabapentin is generally well tolerated. In clinical trials the most common adverse events affected the central nervous system and included somnolence $(24.4 \%)$, dizziness $(20.3 \%)$ and ataxia (17.4\%; Ramsay, 1994). These side-effects tend to be mild and transient (Sussman, 1997). In trials, approximately $7 \%$ of patients on gabapentin withdrew, compared with $3 \%$ of patients on placebo (Ramsay, 1994). Gabapentin does not cause allergic rashes (Anonymous, 1994). Although, like lamotrigine it has been reported to induce mania (Short \& Cooke, 1995; Nassir Ghaemi et al, 1998).

In addition to a weight loss of up to $7 \%$, the most common side-effects of topiramate are dizziness (32\%), fatigue $(29 \%)$, drowsiness $(24 \%)$ and mental slowing (23\%; Anonymous, 1996b).

\section{Drug interactions}

Lamotrigine and gabapentin have no clinically significant effects on P450 hepatic enzymes (Sussman, 1997), and therefore do not interfere with the metabolism of oral contraceptives (Richens, 1994; Elwes \& Binnie, 1996). Lamotigrine has been reported to inhibit epoxide hydrolase increasing carbamazepine epoxide levels, an active metabolite of carbamazepine. and causing diplopia and dizziness (Warner et al, 1992).

Valproate competitively inhibits the glucuronidation of lamotrigine (Elwes \& Binnie, 1996), approximately doubling lamotrigine's half-life to 70 hours (Association of the British Pharmaceutical Industry, 1997). Thus, in a patient taking valproate, lamotrigine should be commenced at a dose of $25 \mathrm{mg}$ on alternate days and increased gradually. It therefore takes six weeks to reach a therapeutic dose of $150 \mathrm{mg}$ daily. Carbamazepine approximately doubles lamotrigine's rate of metabolism (Gilman, 1995).

Unlike lamotrigine, gabapentin has no clinically significant drug interactions with carbamazepine or valproate (Elwes \& Binnie, 1996). Gabapentin has a relatively simple dosing schedule; the starting dose is $\mathbf{3 0 0} \mathrm{mg}$ daily: this can be increased by $300 \mathrm{mg}$ every day to a maximum of $2400 \mathrm{mg}$ (Anonymous, 1994). 
Carbamazapine decreases topiramate plasma concentrations (Doose et al, 1994). Topiramate reduces the effectiveness of combined oral contraceptives (Anonymous, 1996b).

\section{Discussion}

Currently, there is less evidence supporting the use of the newer anticonvulsants in bipolar disorders compared with established agents. Furthermore, there are no comparative studies. The newer anticonvulsants should, therefore, be considered third- or fourth-line agents. Of the four agents, there is more evidence supporting the use of lamotrigine and gabapentin.

Lamotrigine has been suggested as an effective treatment for the depressed phase of bipolar illness, but with a reduced risk of causing mania compared with standard antidepressants (Kusumakar \& Yatham, 1997). Lamotrigine has, however, been reported to induce mania in patients with a bipolar disorder and depression (Sporn \& Sachs, 1997; Kotler \& Matar, 1998).

Lamotrigine has also been concluded to have equivalent mood stabilisation properties, but stronger antidepressant properties than lithium (Kusumakar \& Yatham, 1997). The studies have. however, failed to show any effectiveness in the manic phase. A controlled trial is required to determine whether lamotrigine is a more effective antidepressant, or whether patients are simply switching into manic states.

One of the more controlled studies appears to suggest that gabapentin is particularly effective in treating bipolar disorder type II and bipolar disorder NOS, however, this requires confirmation in larger controlled studies. The dose of gabapentin required also needs to be clarified.

The current results suggest that topiramate may be more effective in treating manic rather than depressive symptoms. Side-effect profile also influences the choice of mood stabiliser. The newer anticonvulsants are generally well tolerated and there is no blood-level monitoring requirement. Unlike lamotrigine, gabapentin does not cause a potentially serious rash and rapid dose escalation is possible. One possible advantage of topiramate is that it causes weight loss.

\section{Summary}

Lamotrigine, gabapentin, topiramate and tiagabine are increasingly being used as mood stabilisers. Unfortunately, there are no controlled trials confirming efficacy. The current data are in the form of case reports and openlabel trials, where there is the potential for falsepositive results due to bias, placebo effects and random variability. Furthermore, patients were usually taking other medication and in some studies there is a high drop out rate.

From the current evidence, the newer anticonvulsants cannot be recommended except in patients resistant to standard therapies. If controlled clinical trials confirm effectiveness, these agents may prove useful additional mood stabilisers.

\section{References}

AMERICAN PSYChiATRIC ASSOCLATION (1994) Diagnostic and Statistical Manual of Mental Disorders (4th edn) (DSMIV). Washington, DC: APA.

ANONYMOUS (1994) Gabapentin - a new antiepileptic drug. Drugs \& Therapeutics Bulletin, 32, 29-30.

- (1996a) Reminder: Lamotrigine and serious skin reactions. Current Problems in Pharmacovigilance, 22, 12.

- (1996b) Topiramate - add-on drug for partial selzures. Drugs \& Therapeutics Bulletin. 34. 62-64.

ASSOCIATION OF THE BRTTSH PHARMACEUTICAL INDUSTRY (1997) Data Sheet Compendium 1997-1998. London: Datapharm Publications Limited.

BENNET, J., GOLDMAN, W. T. \& SUPPES, T. (1997) Gabapentin for treatment of bipolar and schizoaffective disorders (letter). Journal of Clinical Psychopharmacology. 17, 141-142.

CAlABRese, J. R. Bowden, C. L., SAChs, G. S., et al (1999) A double-blind placebo-controlled study of lamotrigine monotherapy in out-patients with bipolar I depression. Journal of Clinical Psychiatry, 60, 79-88.

DODRIL, C. B., ARNETT, J. L., SHU, V., et al (1998) Effects of tiagabine monotherapy on abilities, adjustment, and mood. Epilepsia, 39. 33-42.

Doose. D. R. WALKER, S. A.. SACHDEO, R., et al (1994) Steady-state pharmacokinetics of tegretol (carbamazepine) and topamax (topiramate) in patients with epllepsy on monotherapy and during combination therapy. Epilepsia, 36 (suppl. 8), S54.

DUNCAN, D. MCCONNEL. H. W. \& TAYLOR, D. (1998) Lamotrigine in bipolar affective disorder. Psychiatric Bulletin, 22. 630-632.

ElWEs, R. D. E. \& BinNIE, C. D. (1996) Clinical pharmacokinetics of newer antiepileptic drugs. Clinical Pharmacokinetics, 30, 403-415.

ENDICOTT, J., SPITZER, R. L.. FLeISS, J. L., et al (1976) The Global Assessment Scale: a procedure for measuring overall severity of psychiatric disturbance. Archives of General Psychiatry, 33, 766-771.

GILMAN, J. T. (1995) Lamotrigine: an anti-eplleptic agent for the treatment of partial seizures. Annals of Pharmacotherapy. 29, 144-151.

GuY, W. (ed.) (1976) ECDEU Assessment Manual for Psychopharmacology. Publication ADM 76-338, pp. 217-222. Rockville. MD: US Department of Health Education and Welfare.

HAMILTON. M. (1960) A rating scale for depression. Joumal of Neurology. Neurosurgery and Psychiatry. 23. 56-62.

- (1967) Development of a rating scale for primary depressive illness. British Journal of Social and Clinical Psychology, 6, 278-296.

KNOLL, J., STEGman, K. \& SUPPES. T. (1998) Clinical experience using gabapentin adjunctively in patients with a history of mania or hypomania. Journal of Affective Disorders, 49, 229-233.

KOTLER, M. \& MATAR, M. A. (1998) Lamotrigine in the treatment of resistant bipolar disorder. Clinical Neuropharmacology, 21, 65-67. 
KUSUMAKAR, V. \& YATHAM. L. N. (1997) An open study of lamotrigine in refractory bipolar depression. Psychiatry Research, 72, 145-148.

MAIDMENT. I. D. (1999) Lamotrigine - an effective mood stabiliser? Annals of Pharmacotherapy. in press.

MARCOTTE, D. (1998) Use of topiramate, a new anti-epileptic as a mood stabiliser. Journal of Affective Disorders, 60. 245-251.

MCELROY, S. L., SOUTULO, C. A., KECK. P. E., et al (1997) A pilot trial of adjunctive gabapentin in the treatment of bipolar disorder. Annals of Clinical Psychiatry. 9, 99-103.

NASSIR GhaEMI, S., KATZOW, J. J.. DESA, S. P., et al (1998) Gabapentin treatment of mood disorders: a preliminary study. Journal of Clinical Psychiatry. B9, 426-429.

OVERALL. J. E. \& GoRHAM, D. R. (1962) The Brief Psychiatric Rating Scale. Psychological Reports, 10, 799-812.

RAMSAY, R. E. (1994) Clinical efficacy and safety of gabapentin. Neurology. 44 (suppl. 5). S23-S30.

RiCHENS, A. (1994) Safety of lamotrigine. Epilepsia, 36 (suppl. 5), S37-S40.

RUSH, A. J., GILES, D. E., SChlesser, M. A., et al (1986) The Inventory for Depressive Symptomatology (IDS): preliminary findings. Psychiatry Research, 18, 65-87.

RYBACK, R. \& RYBACK, L. (1995) Gabapentin for behavioural dyscontrol (letter). American Joumal of Psychiatry. 162. 1399.

-. BRODSKY, L. \& MUNASIF, F. (1997) Gabapentin in bipolar disorder (letter). Journal of Neuropsychiatry, 8, 301.

SCHAFFER, C. B. \& SCHAFFER. L. C. (1997) Gabapentin in the treatment of bipolar disorder (letter). American Joumal of Psychiatry. 164, 291-292.
SHORT, C. \& COOKE, L. (1995) Hypomania induced by gabapentin (letter). British Journal of Psychiatry. 168. $679-680$.

SPORN, J. \& SACHS, G. (1997) The anticonvulsant lamotrigine in treatment-resistant manic-depressive illness. Joumal of Clinical Psychopharmacology, 17, 185-189.

Stanton, S. P., KeсK. P. E. \& MCElroy, S. L. (1997) Treatment of acute mania with gabapentin (letter). American Journal of Psychiatry. 184, 287.

STERKER, M. . BERROUSHOT, J. \& SCHNEIDER, D. (1995) Fata course of toxic epidermal necrolysis under treatment with lamotrigine. International Journal of Pharmacology and Therapeutics, 38, 595-597.

SUSSMAN, N. (1997) Gabapentin and lamotrigine: alternative agents for the treatment of bipolar disorder. Psychopharmacology, 132, 25-42.

Warner, T., PAtsalos, P. N, Prevett, M., et al (1992) Lamotrigine-induced carbamazepine-10,11-epoxide. Epilepsy Research, 11, 147-150.

YounG, R. C., BigGs, J. T., ZiEgleR, V. E., et al (1978) A rating scale for mania: reliability, validity and sensitivity. British Journal of Psychiatry. 135. 429435.

Ian Maidment, Clinical Pharmacist, Pharmacy Department, Hellesdon Hospital, Drayton High Road, Norwich NR6 5BE

\section{Recent Topics from Advances in Psychiatric Treatment: Volume 2 Affective and Non-Psychotic Disorders Edited by Alan Lee}

This book discusses the evidence-based assessment of deliberate selfharm, and covers the special problems of general psychiatric practice when alcohol misuse and severe personality disorder complicate the

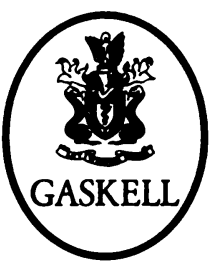
picture. Chapters relating to depressive disorders begin by focusing on opportunities for psychosocial prevention and liaison with primary care. There are comparisons of 'old' and 'new' antidepressants, and reviews of strategies for preventing relapse and recurrence, and managing resistant depression. The special problems of emergency treatment and depression in older patients are identified.

\section{Primary Market}

- Trainees sitting Part II of the Royal College of Psychiatrists Membership Examination

- Specialist Registrars

\section{September 1999, £15.00, Paperback, ISBN 190124217 X}

Royal College of Psychiatrists, Book Sales, 17 Belgrave Square, London, SWIX 8PG, Tel: 01712352351 ext 146, Fax: 0171245 1231, 9.30am - 2.00pm. 